Virginia Commonwealth University vCU Scholars Compass

\title{
Mesopore etching under supercritical conditions - A shortcut to hierarchically porous silica monoliths
}

Martin von der Lehr

Justus-Liebig-Universität Giessen

Kristof Hormann

Philipps-Universität Marburg

Alexandra Höltzel

Philipps-Universität Marburg

See next page for additional authors

Follow this and additional works at: http://scholarscompass.vcu.edu/chem_pubs

Part of the Chemistry Commons

(C) 2017 Elsevier Inc. All rights reserved.

\section{Downloaded from}

http://scholarscompass.vcu.edu/chem_pubs/74

This Article is brought to you for free and open access by the Dept. of Chemistry at VCU Scholars Compass. It has been accepted for inclusion in Chemistry Publications by an authorized administrator of VCU Scholars Compass. For more information, please contact libcompass@vcu.edu. 


\section{Authors}

Martin von der Lehr, Kristof Hormann, Alexandra Höltzel, Lauren S. White, Arved E. Reising, Massimo F. Bertino, Bernd M. Smarsly, and Ulrich Tallarek 


\title{
Mesopore etching under supercritical conditions - A shortcut to hierarchically porous silica monoliths
}

\author{
Martin von der Lehr ${ }^{\text {a }}$, Kristof Hormann ${ }^{b}$, Alexandra Höltzel ${ }^{b}$, Lauren S. White ${ }^{\text {c }}$, \\ Arved E. Reising ${ }^{\mathrm{b}}$, Massimo F. Bertino ${ }^{\mathrm{c}}$, Bernd M. Smarsly ${ }^{\mathrm{a}}$, Ulrich Tallarek ${ }^{\mathrm{b}, *}$ \\ a Institute of Physical Chemistry, Justus-Liebig-Universität Giessen, Heinrich-Buff-Ring 17, 35392 Giessen, Germany \\ b Department of Chemistry, Philipps-Universität Marburg, Hans-Meerwein-Strasse 4, 35032 Marburg, Germany \\ c Department of Physics, Virginia Commonwealth University, Richmond, VA 23060, USA
}

\section{A R T I C L E I N F O}

\section{Article history:}

Received 13 November 2016

Accepted 10 February 2017

Available online 13 February 2017

\section{Keywords:}

Nakanishi process

Surface etching

Supercritical drying

Physical reconstruction

Morphological analysis

\begin{abstract}
A B S T R A C
Hierarchically porous silica monoliths are obtained in the two-step Nakanishi process, where formation of a macro-microporous silica gel is followed by widening micropores to mesopores through surface etching. The latter step is carried out through hydrothermal treatment of the gel in alkaline solution and necessitates a lengthy solvent exchange of the aqueous pore fluid before the ripened gel can be dried and calcined into a mechanically stable macro-mesoporous monolith. We show that using an ethanol-water $(95.6 / 4.4, \mathrm{v} / \mathrm{v})$ azeotrope as supercritical fluid for mesopore etching eliminates the solvent exchange, ripening, and drying steps of the classic route and delivers silica monoliths that can withstand fast heating rates for calcination. The proposed shortcut decreases the overall preparation time from ca. one week to ca. one day. Porosity data show that the alkaline conditions for mesopore etching are crucial to obtain crack-free samples with a narrow mesopore size distribution. Physical reconstruction of selected samples by confocal laser scanning microscopy and subsequent morphological analysis confirms that monoliths prepared via the proposed shortcut possess the high homogeneity of silica skeleton and macropore space that is desirable in adsorbents for flow-through applications.
\end{abstract}

(C) 2017 Elsevier Inc. All rights reserved.

\section{Introduction}

Silica monoliths with interskeleton macropores and intraskeleton mesopores can be prepared by sol-gel processing combined with phase separation and soft templating strategies relying on various structure-directing agents [1]. Many parameters can be addressed to influence the phase separation through spinodal decomposition during the sol-gel process: from the structuredirecting agent chosen as porogen (poor solvents, surfactants, block copolymers, emulsions) over $\mathrm{pH}$ and temperature of the starting solution to type and concentration of the silica precursor. These parameters, which affect the progress of phase separation vs. gelation, influence the morphology of the resulting bi-continuous gel, including the degree of macroscopic separation between silica-rich and solvent-rich phase [2-4]. The chemical surface properties of the monolith can be tailored through selecting

\footnotetext{
* Corresponding author.

E-mail address: tallarek@staff.uni-marburg.de (U. Tallarek).

URL: http://www.uni-marburg.de/fb15/ag-tallarek
}

suitable precursors (co-condensation) or through postsynthesis grafting of functional moieties [5-9].

Following seminal work from the early 1990s [10], Nakanishi and co-workers established a two-step route to macro-mesoporous silica monoliths in which macropore size and volume can be adjusted largely independent from the skeleton thickness and mesopore size [11,12]. Translated into mass-transport relevant terms, the Nakanishi process offers the possibility to adjust the hydraulic permeability (realized by the flow-through macropores) independently from the surface area (realized by the mesopores) and intraskeleton mass transfer resistance (determined by the skeleton thickness) of hierarchically porous silica monoliths [13], which makes them attractive adsorbents for chemical separations $[14,15]$ and solid-liquid catalysis $[16,17]$. Their performance in these processes, and hence the overall process efficiency, profits from a high degree of morphological homogeneity, that is, a narrow distribution of pore size and skeleton thickness [18,19]. These attributes are also advantageous when silica monoliths are used as nanocasting templates [20-22], because the morphological quality of the template limits that of the casted porous medium.

The Nakanishi process starts with a silicon alkoxide and an organic polymer, for example, tetramethoxysilane (TMOS) and 
poly(ethylene glycol) (PEG), in acidic aqueous solution [3]. The sol-gel process yields a macro-microporous gel, which is then submitted to hydrothermal treatment $\left(80-120^{\circ} \mathrm{C}\right)$ under alkaline conditions. The micropores in the gel are widened to mesopores through surface etching with ammonia solution, usually generated in situ through thermal decomposition of urea. Urea can already be added to the reactants for the sol-gel step to ensure a homogeneous distribution in the gel before surface etching takes place [23]. After hydrothermal treatment, the aqueous pore liquid is exchanged for methanol to prevent cracking of the fragile gels during the final calcination step. The gel is immersed for 3-5 days in repeatedly refreshed methanol, during which the pore solvent is exchanged and a ripening process occurs in which the siliceous matrix stiffens and shrinks. The ripened gel is calcined at typically $330-550{ }^{\circ} \mathrm{C}$, during which the structure is dried, residual template removed from the pores (by thermal decomposition of PEG), and condensation of the silica backbone completed. Calcination is approached with a shallow heating ramp (i.e., slowly) to avoid the build-up of capillary stress that would crack the monolithic structure. From starting sol to finished monolith, the Nakanishi process takes about one week, whereby most of the preparation time is taken up by waiting for slow processes to complete (Scheme 1).

The time-limiting step in the Nakanishi process is solvent exchange. The same problem has turned up earlier in aerogel fabrication, where supercritical extraction (SCE), which avoids the build-up of capillary stress due to the absence of liquid-gas interfaces, has long been used as an efficient, quick, and mild method for drying $[24,25]$. SCE drying, however, involves exchanging the pore fluid present after gelation for a suitable SCE solvent $\left(\mathrm{CO}_{2}\right.$ or a low alcohol). For fast aerogel fabrication, the dreaded solvent exchange was circumvented by carrying out the whole synthesis (gelation, aging, drying) under supercritical conditions or by choosing a gelation solvent also suitable for SCE drying. Recent examples of fast (5-6 h) aerogel syntheses include a one-step, reactant-to-aerogel route using supercritical methanol [26] as well as a route where an ethanol-water azeotrope served as gelation and as supercritical drying solvent [27,28].
Although an established drying method for aerogels, SCE has not been employed in the preparation of hierarchically porous silica monoliths, except to prevent cracking under special circumstances. Nakanishi and co-workers [29] used the mild conditions of SCE with $\mathrm{CO}_{2}$ ("cold drying" at $31^{\circ} \mathrm{C}$ ) to obtain exceptionally large silica monoliths (1.1-L volume) with average mesopore diameters $\leq 30 \mathrm{~nm}$. While cold SCE drying prevents cracking, it does not reduce the preparation time, because the low miscibility of water and liquid $\mathrm{CO}_{2}$ requires two complete solvent exchanges: from aqueous pore liquid to methanol to liquid $\mathrm{CO}_{2}$.

In this work, we eliminate the solvent exchange step from the Nakanishi process through carrying out mesopore etching under the conditions of SCE drying. For surface etching, we need a supercritical fluid that supports the thermal decomposition of urea. Between the two obvious candidate solvents for this task, ethanol is preferred over methanol, because methanol is toxic and supercritical methanol is not inert towards silica. In aerogel drying with supercritical methanol, for example, an unwanted alkylation of surface $\mathrm{OH}$ groups and partial dissolution of the silica structure was observed $[25,30]$. Because the pores hold water from the sol-gel processing step, the SCE fluid is an ethanol-water azeotrope (95.6/ $4.4, \mathrm{v} / \mathrm{v}$ ) with a critical temperature of $265^{\circ} \mathrm{C}$. The main task of the supercritical ethanol-water azeotrope (SCEWA) is to provide the conditions for surface etching and SCE drying, but the high temperature and fast mass transport properties of the supercritical fluid are additionally expected to accelerate all involved processes. We thus propose the SCEWA step (Scheme 1) to speed up mesopore formation and ripening, eliminate solvent exchange, and yield dry monoliths that can withstand fast calcination.

\section{Experimental}

\subsection{Chemicals}

TMOS was purchased from Acros Organics (Geel, Belgium), PEG $\left(M_{\mathrm{n}}=10^{4}\right)$ and urea came from Merck Millipore (Darmstadt, Germany). Acetic acid, glycerol, and octadecyltrimethoxysilane were

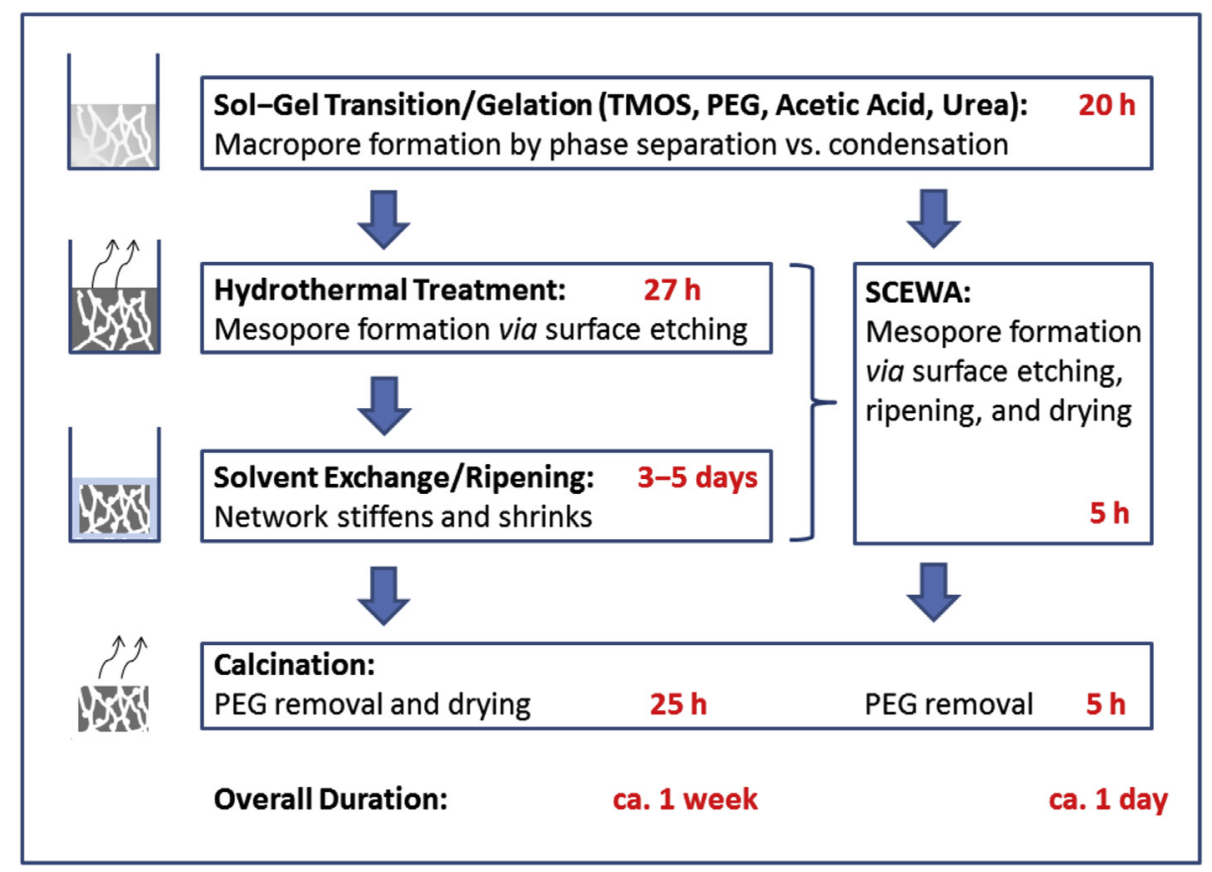

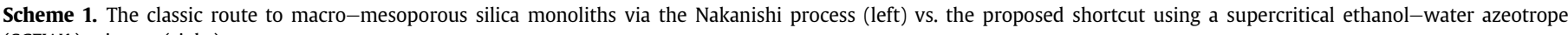
(SCEWA) mixture (right). 
obtained from Sigma-Aldrich (Buchs, Switzerland, and Taufkirchen, Germany). The fluorescent dye Bodipy 493/503 was supplied by Life Technologies (Darmstadt, Germany). Dimethylsulfoxide (DMSO), methanol (HPLC grade), and $\mathrm{NaOH}$ (99\%, p.a.) came from Carl Roth (Karlsruhe, Germany). Ethanol (190 proof) was purchased from Pharmco-Aaper (Brookfield, CT). HPLCgrade water was obtained from a Milli-Q gradient purification system (Millipore, Bedford, MA).

\subsection{Preparation of monolith samples}

We first prepared a set of eight gel pieces by following an upscaled version of an established procedure [23]. A mixture of $5.5 \mathrm{~g}$ PEG $(0.55 \mathrm{mmol})$ and $4.5 \mathrm{~g}$ urea $(91.5 \mathrm{mmol})$ in $50 \mathrm{ml}$ acetic acid $\left(0.01 \mathrm{~mol} \mathrm{l}^{-1}\right)$ was stirred vigorously for $10 \mathrm{~min}$ at room temperature and for $15 \mathrm{~min}$ at $0{ }^{\circ} \mathrm{C}$ (ice bath). After addition of $28 \mathrm{~mL}$ TMOS ( $189.4 \mathrm{mmol})$ the mixture was stirred for $25 \mathrm{~min}$ at $0{ }^{\circ} \mathrm{C}$ to induce phase separation by spinodal decomposition of the system. The resulting sol was filled in $10-\mathrm{ml}$ portions into $15-\mathrm{ml}$ plastic vials and allowed to gel for $16 \mathrm{~h}$ at $25{ }^{\circ} \mathrm{C}$ (water bath).

The SCEWA step was carried out for each gel piece separately. We used a 2-L capacity autoclave with pressure gauge and heating mantle (Parr Instrument Company, Moline, IL). To prevent contact between gel piece and autoclave bottom and to ensure complete immersion of the gel piece in the reaction mixture, the gel piece was placed on a footed stainless steel mesh that was then lowered into the autoclave containing the reaction mixture. The reaction mixture was $45 \mathrm{~g}$ urea dissolved in $0.5 \mathrm{l}$ ethanol $\left(1.5 \mathrm{~mol}^{-1}\right.$ urea) for samples 1 and 3, $4.5 \mathrm{~g}$ urea dissolved in 0.51 ethanol ( $0.15 \mathrm{~mol} \mathrm{l}^{-1}$ urea) for samples 2 and $4-8$, plus solid $\mathrm{NaOH}$ in the amounts given in Table 1 for samples $3-8$. The system was heated over $3 \mathrm{~h}$ to supercritical conditions, which were maintained for 20 min before venting and cooling. Fig. 1 shows a typical heating ramp and the resulting pressures in the system. The SCEWAprepared silica monoliths had a diameter of $1.1 \mathrm{~cm}$ and a length of $2.7 \mathrm{~cm}$.

Sample 4 was subsequently calcined for $4 \mathrm{~h}$ at $550{ }^{\circ} \mathrm{C}$, reached by applying a heating rate of $8.8^{\circ} \mathrm{C} / \mathrm{min}$.

Hazard note. Because the molar volume of supercritical ethanol is about four times larger than that of liquid ethanol, excess solvent can raise the pressure level beyond the permitted maximum value. The total filling level of the autoclave should therefore be cautiously calculated to respect the permitted maximum pressure in the vessel. The autoclave should be vented into a water bath to prevent the release of highly flammable ethanol vapors into the room. Only stainless steel must be used inside the autoclave, as other metals can react with the solvent, leading to hazardous pressure increases. It should also be noted that using high urea concentrations

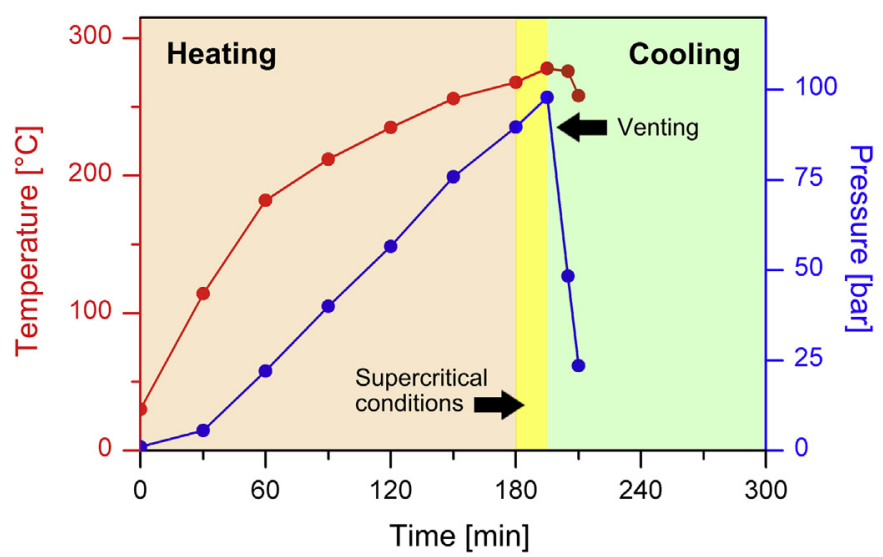

Fig. 1. Progress of temperature (red) and pressure (blue) in the autoclave during the SCEWA step. (For interpretation of the references to colour in this figure legend, the reader is referred to the web version of this article.)

$\left(1.5 \mathrm{~mol} \mathrm{l}^{-1}\right)$ in the reaction mixture led to formation of urea plugs under the piston of the manometer.

\subsection{Characterization}

Monolith samples were examined by a scanning electron microscope (Smart SEM MERLIN, Carl Zeiss, Jena, Germany) using a fractured surface. Samples were platinum-coated by a sputter coater (HHV Scancoat Six, Boc Edwards, Kirchheim, Germany).

Nitrogen adsorption-desorption isotherms were determined on an automated gas adsorption station (QUADRASORB evo, Quantachrome Corporation, Boynton Beach, FL). Samples were evacuated for $16 \mathrm{~h}$ at $120{ }^{\circ} \mathrm{C}$ prior to analysis at $77 \mathrm{~K}$. Isotherms were measured up to $p / p_{0}=0.95$. Pore size distributions were derived from the adsorption branches using the non-local density functional theory (NLDFT) method with a cylindrical pore model.

Mercury intrusion porosimetry (MIP) was run on Pascal 140/440 porosimeters (Thermo Fisher Scientific, Rodano, Italy). Intrusion measurements were performed over a pressure range of 0.15-400 MPa in small increments. Pore size distributions were calculated according to the Washburn equation, assuming a mercury surface tension of $0.48 \mathrm{~N} / \mathrm{m}$ and a contact angle of $140^{\circ}$. A blank correction file was used to compensate for mercury compressibility and temperature effects.

Thermogravimetric analysis with mass spectrometric detection (TGA-MS) was conducted at a heating rate of $5^{\circ} \mathrm{C} / \mathrm{min}$ up to $800{ }^{\circ} \mathrm{C}$ on a STA 409 PC system (Netzsch, Selb, Germany) coupled to a QMG 201 quadrupole mass spectrometer (Balzers Instruments, Balzers, Liechtenstein). Fourier-transform infrared (FT-IR) spectra were

Table 1

Preparation conditions and porosity data for SCEWA-prepared silica monoliths.

\begin{tabular}{|c|c|c|c|c|c|c|c|}
\hline Sample & Conditions & $S_{\text {BET }}\left[\mathrm{m}^{2} / \mathrm{g}\right]^{\mathrm{a}}$ & $d_{\text {meso }}[\mathrm{nm}]^{\mathrm{a}, \mathrm{b}}$ & $V_{\text {meso }}\left[\mathrm{cm}^{3} / \mathrm{g}\right]^{\mathrm{a}, \mathrm{b}}$ & $d_{\text {macro }}[\mu \mathrm{m}]^{\mathrm{c}}$ & $V_{\text {macro }}\left[\mathrm{cm}^{3} / \mathrm{g}\right]^{\mathrm{c}}$ & Cracking \\
\hline 1 & $1.5 \mathrm{M}$ urea, no $\mathrm{NaOH}$ & 284 & $7(6)$ & $0.61(0.60)$ & 3.33 & 1.53 & Yes \\
\hline 2 & $0.15 \mathrm{M}$ urea, no $\mathrm{NaOH}$ & 267 & $9(9)$ & $0.73(0.57)$ & 3.25 & 1.68 & Yes \\
\hline 3 & $1.5 \mathrm{M}$ urea, $0.02 \mathrm{~g} \mathrm{NaOH}$ & 244 & $9(9)$ & $0.67(0.54)$ & 3.54 & 1.60 & No \\
\hline 4 & $0.15 \mathrm{M}$ urea, $0.02 \mathrm{~g} \mathrm{NaOH}$ & 180 & $11(13)$ & $0.71(0.63)$ & 3.33 & 1.42 & No \\
\hline 5 & $0.15 \mathrm{M}$ urea, $0.1 \mathrm{~g} \mathrm{NaOH}$ & 111 & $14(16)$ & $0.70(0.50)$ & 3.49 & 1.23 & No \\
\hline 6 & $0.15 \mathrm{M}$ urea, $0.2 \mathrm{~g} \mathrm{NaOH}$ & 117 & $14(16)$ & $0.67(0.56)$ & 3.56 & 1.67 & No \\
\hline 7 & $0.15 \mathrm{M}$ urea, $0.3 \mathrm{~g} \mathrm{NaOH}$ & 92 & $17(28)$ & $0.69(0.51)$ & 3.58 & 1.09 & No \\
\hline 8 & $0.15 \mathrm{M}$ urea, $0.4 \mathrm{~g} \mathrm{NaOH}$ & 46 & $29(70)$ & $0.50(0.17)$ & 3.56 & 1.33 & No \\
\hline 4 calcined & to $550{ }^{\circ} \mathrm{C}$ in $1 \mathrm{~h}$, at $550{ }^{\circ} \mathrm{C}$ for $4 \mathrm{~h}$ & 252 & $12(14)$ & $0.86(0.77)$ & 3.26 & 2.01 & No \\
\hline
\end{tabular}

\footnotetext{
a Calculated from nitrogen sorption data.

b Values in brackets calculated from MIP data.

c Calculated from MIP data.
} 
recorded at wavelengths from 4000 to $400 \mathrm{~cm}^{-1}$ on a Bruker FT-IRIFS 85 spectrometer (Karlsruhe, Germany) using the $\mathrm{KBr}$ pellet technique.

\subsection{Physical reconstruction by confocal laser scanning microscopy (CLSM)}

For CLSM imaging, a ca. 1-mm thick disk was cut from samples 2, 3 , and 4 each. The disks were first treated with octadecyltrimethoxysilane for a chemically-bonded, hydrophobic modification of the silica surface, and then stained by adsorption of the fluorescent dye Bodipy 493/503 onto the hydrophobic surface [31]. The pore fluid in the stained disks was then exchanged for a glycerol/DMSO/water $(70 / 19 / 11, \mathrm{v} / \mathrm{v} / \mathrm{v})$ mixture that matches the refractive index of the silica skeleton at the sodium $\mathrm{D}$ line $\left(n_{\mathrm{D}}=1.4582\right)$. The matching liquid was also used for embedding and immersion of the sample in the CLSM measurements. Matching the optical dispersion behavior of the silica monoliths allowed imaging into the bulk of a sample. CLSM images were acquired on a TCS SP5 II confocal microscopy system equipped with a HCX PL APO $63 \times / 1.3$ GLYC CORR CS $\left(21^{\circ} \mathrm{C}\right)$ glycerol immersion objective lens (Leica Microsystems, Wetzlar, Germany). A stained monolith disk saturated with matching liquid was placed into the matching-liquid filled well of a custom-made sample holder. The separation of embedding and immersion liquid by a 'type 0' cover slip (Gerhard Menzel, Braunschweig, Germany) minimized spherical aberration effects. Stacks of 8-bit grayscale images (4096 $\times 4096$ pixels) were acquired with a resolution of $30 \mathrm{~nm}$ (lateral) $\times 126 \mathrm{~nm}$ (axial) in compliance with the Nyquist criterion. Images were restored by denoising, photobleaching correction, and deconvolution prior to segmentation into binary images of solid (silica phase) and void pixels (macropore space). The segmented images were then used to generate physical reconstructions of the monolith samples at macropore resolution.

\subsection{Chord length distribution (CLD) analysis of physically reconstructed samples}

Physically reconstructed monolith samples were subjected to CLD analysis to obtain quantitative measures for the homogeneity of silica phase (solid, with the unresolved mesopores) and macropore (void) space [32]. For a CLD of the macropore space, random points were generated in the void area of an image stack. From each of these points 32 vectors were projected in angularly equispaced directions. Vectors either hit the skeleton or they projected out of the image and were discarded. Points of origin were generated until $10^{6}$ chords (the sum of the absolute lengths of an opposed pair of vectors) were collected into a histogram (CLD). A CLD of the silica skeleton was obtained by generating points of origin in the solid phase and proceeding analogously. In materials with correlated disorder (contrary to materials with Debye-randomness or highly ordered materials), the CLDs can be described by a $k$-gamma function [18]

$f\left(l_{\mathrm{c}}\right)=\frac{k^{k}}{\Gamma(k)} \frac{l_{\mathrm{c}}^{k-1}}{\mu^{k}} \exp \left(-k \frac{l_{\mathrm{c}}}{\mu}\right)$

where $l_{c}$ is the chord length, $\Gamma$ the gamma function, $\mu$ the mean of the distribution, and $k=(\mu / \sigma)^{2}$ is the squared ratio of the mean to the standard deviation of the distribution. The $k$-gamma function was fitted to the CLDs using the Levenberg-Marquardt algorithm [31].

\section{Results and discussion}

\subsection{Mesopore etching with the SCEWA step}

The first task in establishing the SCEWA step consisted of finding the proper conditions for mesopore etching. We used a set temperature program for the SCEWA step (Fig. 1) and varied the composition of the reaction mixture in the autoclave between individual samples. The eight gel samples submitted to the SCEWA step were synthesized as one charge in the sol-gel step, so that variations between the samples detected after the SCEWA step should reflect the effect of the reaction mixture compositions used. All samples were characterized after the SCEWA step by nitrogen sorption and MIP analysis. This was possible because the samples were dry and stable, in contrast to monolith samples prepared via the classic route, which can only be analyzed after calcination. Table 1 summarizes our synthetic efforts with the SCEWA step by linking the composition of the reaction mixture used for mesopore etching with the porosity data for the prepared monolith samples as determined from nitrogen sorption and MIP measurements, namely the Brunauer-Emmett-Teller (BET) surface area $\left(S_{\mathrm{BET}}\right)$, mean macropore and mesopore size $\left(d_{\text {macro }}, d_{\text {meso }}\right)$, and macropore and mesopore volume $\left(V_{\text {macro }}, V_{\text {meso }}\right)$. Fig. 2 shows the nitrogen sorption isotherms for the SCEWA-prepared samples and the pore size distributions derived from NLDFT analysis of the adsorption branch together with the MIP-derived pore size distributions. The MIP data prove that all SCEWA-prepared silica monoliths possess a hierarchical pore space. The mean interskeleton pore size of samples 1-8 falls (as intended) within the narrow range of $d_{\text {macro }}=3.25-3.58 \mu \mathrm{m}$, whereas the MIP-determined mean size for the intraskeleton pores varies between 6 and $70 \mathrm{~nm}$. These data show that the SCEWA step supports, like hydrothermal treatment, a variation of the intraskeleton pore size independent from the interskeleton pore size, and that the reaction mixture composition has a huge influence on the intraskeleton pore size.

As explained in the Introduction, surface etching is performed by ammonia solution generated through thermal decomposition of urea, which is usually already added to the starting sol and thus incorporated in the gel. Nevertheless, we consistently used urea in the reaction mixture to prevent that in-situ generated ammonia diffuses from the small gel piece out into the large autoclave volume and is lost for mesopore etching. We first tried a pure urea solution as the reaction mixture, evaluating two different concentrations: the urea concentration in the starting solution $\left(1.5 \mathrm{~mol} \mathrm{l}^{-1}\right.$, sample 1) and a ten-fold reduced concentration $\left(0.15 \mathrm{~mol} \mathrm{l}^{-1}\right.$, sample 2). Samples 1 and 2 had rather small mesopores according to the nitrogen sorption data $\left(d_{\text {meso }}=7\right.$ or $\left.9 \mathrm{~nm}\right)$ with narrow size distributions, but were both cracked. Because the capillary pressure that leads to cracking rises with decreasing pore diameter, we concluded from the experience with samples 1 and 2 that we needed to widen the mesopores by increasing the concentration of the active etching agent, which is the hydroxide anion. We tried this by adding a small amount of solid $\mathrm{NaOH}$ as booster chemical to the reaction mixture. (The solid form of $\mathrm{NaOH}$ was chosen over $\mathrm{NaOH}$ solution to avoid the presence of excess water in the autoclave.) Samples 3 and 4 were prepared with a reaction mixture of $0.02 \mathrm{~g}$ solid $\mathrm{NaOH}$ and 1.5 or $0.15 \mathrm{~mol}^{-1}$ urea solution, respectively. Both samples were crack-free and fulfilled our target criteria for the mesopore space; the nitrogen sorption data for samples 3 and 4 show narrow mesopore size distributions (ranging ca. from 6 to $21 \mathrm{~nm}$ ) around mean values of $d_{\text {meso }}=9-11 \mathrm{~nm}$ and $\mathrm{H} 2$ hysteresis behavior [33]. Contrary to the presence of solid $\mathrm{NaOH}$ in the reaction mixture, using a 10-times lower urea concentration than in the starting sol had no decisive effect on the prepared samples. With the high urea concentration in the reaction mixture (samples 1 and 

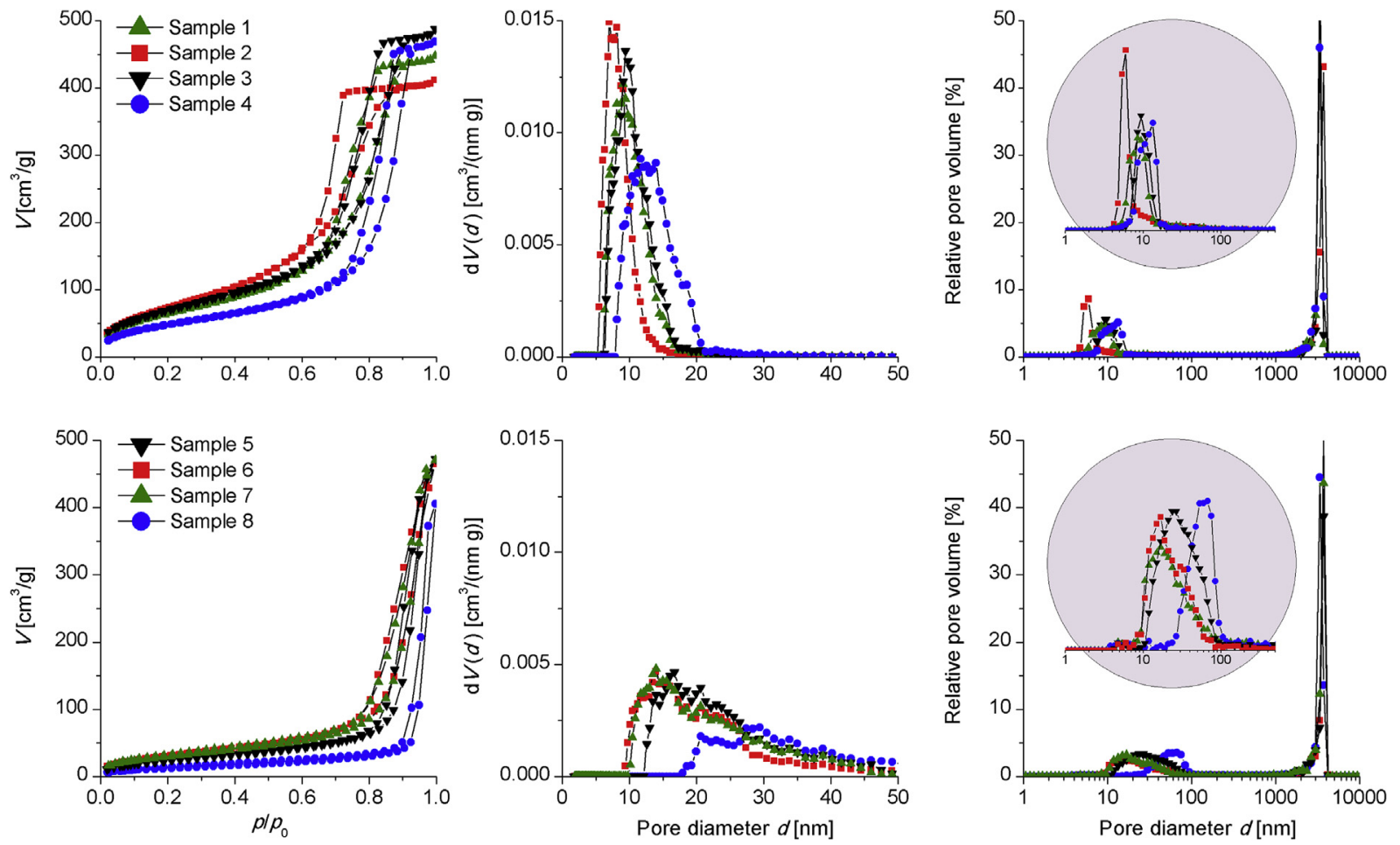

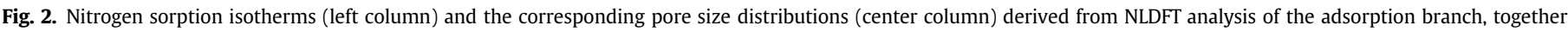
with MIP-derived pore size distributions (right column) for SCEWA-prepared silica monoliths (cf. Table 1).

3), we had encountered technical difficulties through formation of urea plugs in the autoclave. A lower urea concentration in the reaction mixture shifted the mesopore size distributions of the prepared samples to slightly higher values (compare samples 2 and 4 with samples 1 and 3, respectively, in Fig. 2), avoided urea plug formation in the autoclave, and was thus preferable.

After samples 1-4 had revealed the addition of $\mathrm{NaOH}$ to the reaction mixture as critical to the success of the SCEWA step, we next investigated how much $\mathrm{NaOH}$ could be added before it had a negative effect. Samples 5-8 were prepared with $0.15 \mathrm{~mol} \mathrm{l}^{-1}$ urea solution and $0.1-0.4 \mathrm{~g}$ added $\mathrm{NaOH}$. All samples were crack-free, but did not fulfill the target criteria for the mesopore space. The nitrogen sorption isotherms for samples 5-8 (Fig. 2) indicate the presence of intraskeleton pores beyond the mesopore range $(>50 \mathrm{~nm})$, which means that the derived pore size distributions underestimate the true pore size, as proven by comparison with the MIP-derived values. The MIP data for samples $5-8$ show very wide size distributions for the intraskeleton pores, ranging from 10 to $100 \mathrm{~nm}$. Sample 8, in particular, has more intraskeleton pores in the sub- $\mu \mathrm{m}$ range than in the mesopore regime. Samples 5 and 6 have an acceptable mean mesopore size around $15 \mathrm{~nm}$, but contain too many pores with sizes $>30 \mathrm{~nm}$ to be acceptable. Overall, the porosity data for samples $1-8$ show that using small amounts of solid $\mathrm{NaOH}(0.02 \mathrm{~g})$ in addition to urea solution as the reaction mixture for the SCEWA step is crucial to obtain crack-free monoliths with narrow mesopore size distributions around $d_{\text {meso }} \approx 10 \mathrm{~nm}$.

\subsection{Calcination after the SCEWA step}

Having identified the proper alkaline conditions for the SCEWA step $\left(0.15 \mathrm{~mol} \mathrm{l}^{-1}\right.$ urea solution $+0.02 \mathrm{~g}$ solid $\left.\mathrm{NaOH}\right)$, the corresponding sample 4 (Fig. 3) was selected for further processing. We judged the morphological quality of the SCEWA-prepared monolith by comparison with Chromolith rods, which are silica monoliths in analytical column format developed for high-performance liquid chromatography (HPLC) of small molecules. The commercially available Chromolith rods are the benchmark because their porosity data and their performance as adsorbents in

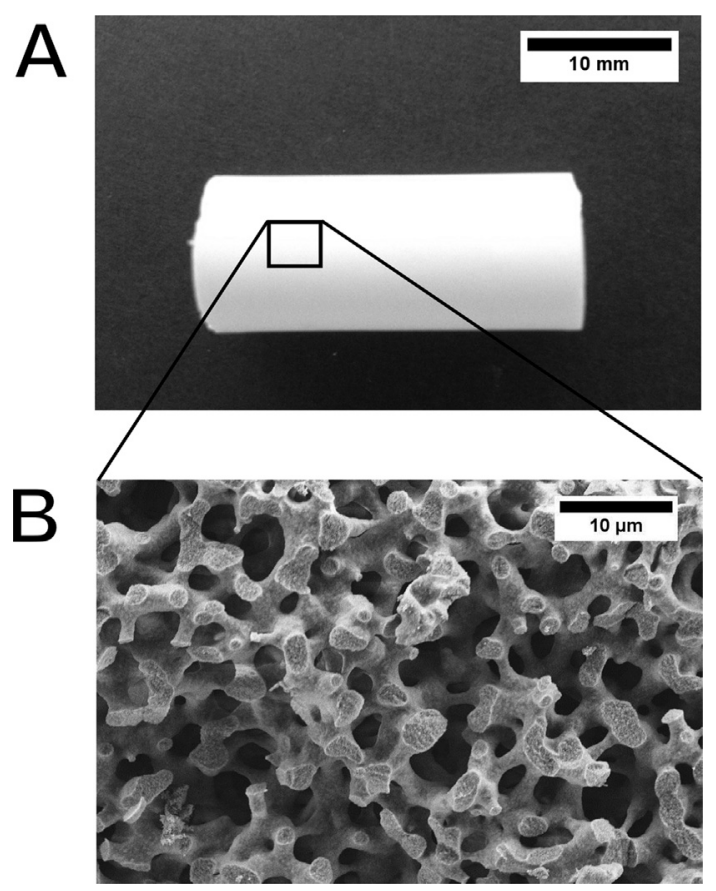

Fig. 3. (A) A crack-free silica monolith after the SCEWA step (sample 4, cf. Table 1). (B) The SEM image shows the hierarchical pore space, realized by interskeleton macropores $\left(d_{\text {macro }}=3.33 \mu \mathrm{m}\right)$ and intraskeleton mesopores $\left(d_{\text {meso }}=11 \mathrm{~nm}\right)$. 
chromatographic separations are well documented [14,15,31], and their morphology is thoroughly characterized through the application of statistical analysis methods to physically reconstructed samples and direct numerical simulations of flow and mass transport in the physically reconstructed samples [31,34-36]. Compared with Chromolith rods [34], sample 4 has a comparatively small BET surface area and macropore volume, because the template (PEG) was not removed from the pore space in the SCEWA step. In the classic route, calcination follows after solvent exchange and serves the dual purpose of template removal and drying (Scheme 1). After solvent exchange monoliths are wet and fragile, necessitating a shallow heating ramp to calcination to avoid cracking. After the SCEWA step, in contrast, monoliths are practically dry and mechanically stable (Fig. 3), so that cracking need not be feared. Sample 4 tolerated a steep heating rate of $8.8^{\circ} \mathrm{C} / \mathrm{min}$, so that the calcination temperature of $550{ }^{\circ} \mathrm{C}$ was reached in only $1 \mathrm{~h}$. The suitability of the chosen calcination temperature was informed by previous experience with the classic route and validated by TGA-MS (Fig. S1). PEG decomposition products were not detected at temperatures $<460{ }^{\circ} \mathrm{C}$; apparently, the decomposition and evaporation of PEG trapped in the pore space of the monolith requires higher temperatures than the thermal decomposition of unconfined PEG. Striving for a short calcination period in line with the short SCEWA step, we found that $4 \mathrm{~h}$ sufficed to remove the template from the pore space of the monolith. A comparison of the FT-IR spectra of sample 4 acquired after the SCEWA step and after calcination (Fig. S2) shows that the bands caused by stretch vibrations of the PEG methylene groups have vanished after calcination. The porosity data for the calcined sample 4 (Table 1 , nitrogen sorption and MIP data in Fig. S3) reveal that calcination has increased the macropore volume of the SCEWA-prepared monolith from 1.42 to $2.01 \mathrm{~cm}^{3} / \mathrm{g}$, the mesopore volume from 0.71 to $0.86 \mathrm{~cm}^{3} / \mathrm{g}$, and the BET surface area from 180 to $252 \mathrm{~m}^{2} / \mathrm{g}$. For first- and second-generation Chromolith rods, a BET surface area of $265 \mathrm{~m}^{2} / \mathrm{g}$, a macropore volume of 2.15 and $2.33 \mathrm{~cm}^{3} / \mathrm{g}$, and a mesopore volume of 0.73 and $0.90 \mathrm{~cm}^{3} / \mathrm{g}$, respectively, had been determined [34]. According to these data, the calcined sample 4 is comparable to Chromolith rods in BET surface area and pore volume.

\subsection{Morphological quality of SCEWA-prepared silica monoliths}

To assess the morphological quality of SCEWA-prepared silica monoliths and specifically to investigate whether mesopore formation under the chosen supercritical conditions affects the preformed macropore space of a monolith, samples 2,3 , and 4 were selected for physical reconstruction at macropore resolution by CLSM. Sample 4 meets the target conditions fully, and samples 2 and 3 are closest to sample 4 regarding the alkaline conditions used in the SCEWA step (Table 1). The CLSM-reconstruction of sample 3 (Fig. 4A) shows a silica skeleton (containing the unresolved mesopores) laced by a network of open macropores, as known from previous reconstructions of silica monoliths prepared via the Nakanishi process [31,34-37]. The macroporosity (interstitial void volume fraction) of the reconstructed samples was determined as the number of voxels assigned to the void space divided by the total number of voxels in a reconstructed volume. With values of $64.9 \%$ (sample 2), 63.4\% (sample 3), and 65.9\% (sample 4), the three samples are (as intended) nearly identical in their macroporosity, which confirms that the SCEWA step does not interfere with the reproducibility of this important parameter. For Chromolith rods, very similar macroporosity values of $65-68 \%$ have been determined from physical reconstructions [31,34-36].

The homogeneity of macropore space and silica skeleton of the SCEWA-prepared monolith samples was determined by CLD
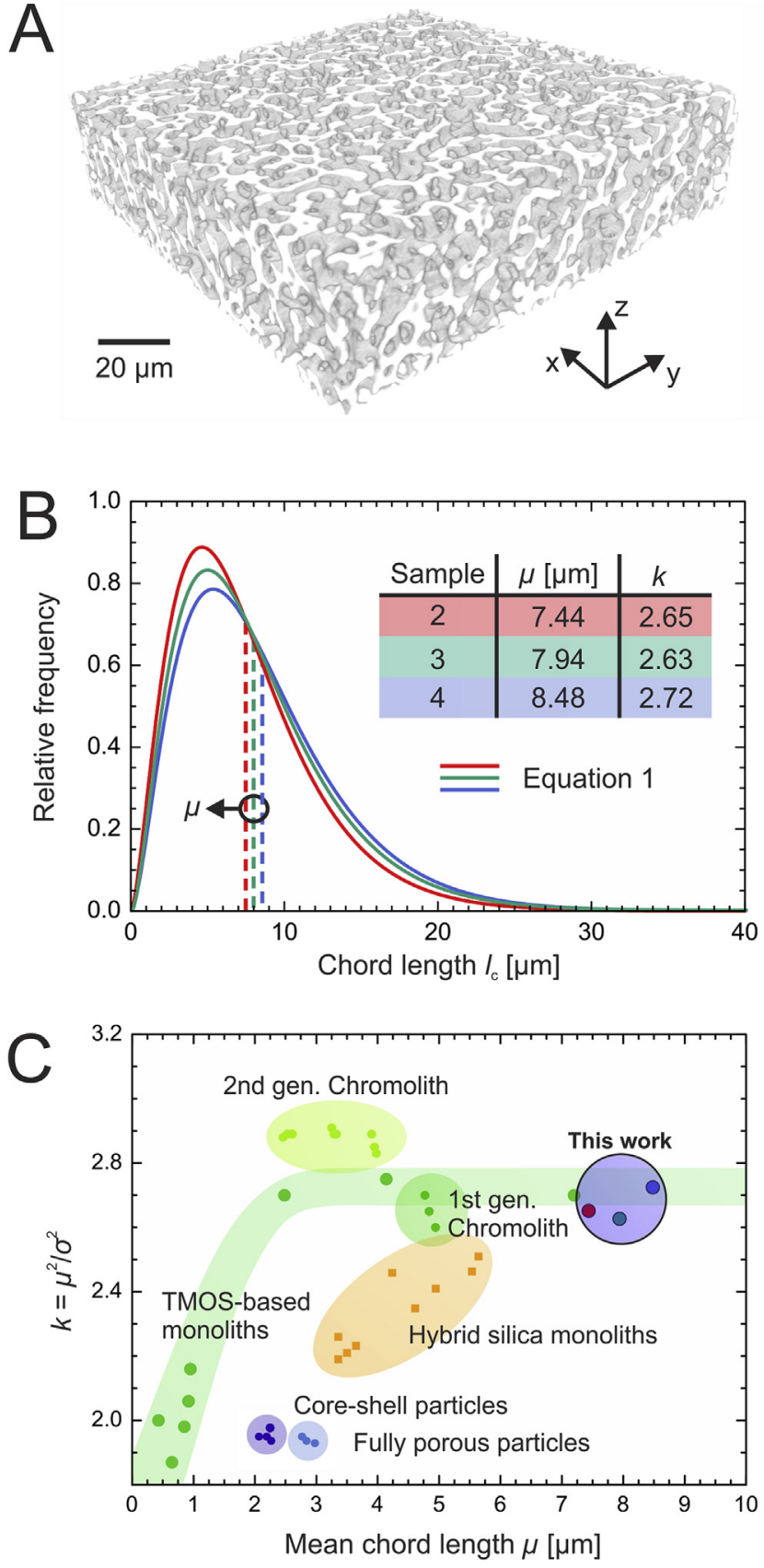

Fig. 4. (A) Physical reconstruction of a silica monolith after the SCEWA step (sample 3 cf. Table 1). 250 CLSM images acquired in $z$-direction cover a physical volume of $120 \mu \mathrm{m} \times 120 \mu \mathrm{m} \times 31 \mu \mathrm{m}(x \times y \times z)$. The silica skeleton (white) contains the unresolved mesopores. (B) Best fits of the $k$-gamma function, Eq. (1), to the CLDs obtained for the reconstructed macropore space of SCEWA-prepared samples 2,3 , and 4 (cf. Table 1). The fits return values for the mean chord length $\mu$ and the pore size homogeneity parameter $k$. (C) Comparison of $\mu$ - and $k$-values determined for SCEWAprepared samples 2,3 , and 4 with values reported for silica-based adsorbent structures (monoliths and particulate packings). Adapted from Müllner et al. [18] with permission from the Center National de la Recherche Scientifique (CNRS) and The Royal Society of Chemistry.

analysis, which differs conceptually from the type of analysis used for deriving pore size distributions from MIP data. The latter assumes an idealized macropore space of well-defined, cylindrical pores, whose diameters can be derived and their mean value ( $\left.d_{\text {macro }}\right)$ calculated. CLD analysis makes no assumptions about the geometry of the constituting elements of a porous medium, but 
provides an accurate description through scanning the solid-void borders by chords of variable length, delivering a histogram (CLD) of all possible straight distances within void space or solid phase. Fitting a CLD to the $k$-gamma function, Eq. (1), delivers values for the mean chord length $\mu$ and for the homogeneity parameter $k$ (larger $k$-values indicate more homogeneous structures). The homogeneity of macropore space and silica skeleton is important for the residence time distribution of solutes in an adsorbent used for solid-liquid catalysis or chromatographic separations. Solutes traverse the macropore space through advective flow and the mesoporous skeleton through diffusion. A homogeneous macropore space reduces the velocity bias between neighboring flow channels and thus the "backmixing" of solutes due to flow heterogeneities. A homogeneous silica phase supports similar diffusion path lengths for solutes. A low degree of flow heterogeneity and similar diffusion path lengths contribute to a narrow residence time distribution of solutes, which is necessary for high process efficiency [19]. Fig. 4B shows the best fits of the $k$-gamma function, Eq. (1), to the macropore CLDs of samples 2, 3, and 4 (solid lines), together with the values extracted for $\mu$ and $k$ (inset). The reconstructed macropore spaces are characterized by $\mu$-values of $7.44-8.48 \mu \mathrm{m}$ and $k$-values of $2.63-2.72$. These values are put into perspective by comparison with data from previous reconstructions of classically prepared silica monolith rods and alternative silica-based adsorbent structures (Fig. 4C). The green shading in Fig. 4C sums up the experience made with silica monoliths so far: they have high $k$-values $(2.6-2.8)$ when their $\mu$ values are in the $>2 \mu \mathrm{m}$ range, that is, the macropore space is highly homogeneous as long as the macropore size does not reach the sub$\mu \mathrm{m}$ range [37]. The high macropore space homogeneity of silica monoliths is one of their main advantages over alternative adsorbent structures, namely, packings of core-shell or fully porous particles. The SCEWA-prepared samples are closest to firstgeneration Chromolith rods in macropore space homogeneity. (The high macropore space homogeneity of second-generation Chromolith rods has not yet been observed with monolith samples from academic laboratories.) For the silica phase of samples 2, 3 , and 4 the CLD analysis returned values of $\mu=3.65-3.74 \mu \mathrm{m}$ and $k=3.54-3.62$, reflecting that the SCEWA-prepared samples also match the high skeleton homogeneity $(k=3.37-3.93)$ of Chromolith rods [34]. Judging by the data received from CLD analysis of physically reconstructed samples, the SCEWA-prepared silica monoliths recover the morphological homogeneity of hierarchically porous silica monoliths used as adsorbents in HPLC applications.

\section{Conclusions}

Our results show that carrying out mesopore etching, drying, and ripening simultaneously under supercritical conditions is an efficient way to replace the time-consuming hydrothermal treatment, solvent exchange, and ripening steps of the Nakanishi process without compromising the morphological quality of the prepared silica monoliths. The alkaline conditions used for mesopore etching during the comparatively short SCEWA step are critical to the success of the synthesis: urea as the primary reagent must be supplemented with small amounts of solid $\mathrm{NaOH}$ to obtain crack-free monoliths with narrow mesopore size distributions. Monoliths emerging from the SCEWA step are stable enough for analysis and require only a short calcination period for template removal. With the proposed shortcut, macro-mesoporous silica monoliths can be obtained in $30 \mathrm{~h}$, a time span that encourages variations in synthetic parameters to explore the full potential of the Nakanishi process.

\section{Acknowledgement}

We thank Woldemar Niedenthal for his assistance with the SCEWA experiments.

\section{Appendix A. Supplementary data}

Supplementary data related to this article can be found at http:// dx.doi.org/10.1016/j.micromeso.2017.02.036.

\section{References}

[1] A. Feinle, M.S. Elsaesser, N. Hüsing, Chem. Soc. Rev. 45 (2016) 3377-3399.

[2] J.-H. Smått, S. Schunk, M. Lindén, Chem. Mater. 15 (2003) 2354-2361.

[3] K. Nakanishi, N. Tanaka, Acc. Chem. Res. 40 (2007) 863-873.

[4] K. Kanamori, K. Nakanishi, Chem. Soc. Rev. 40 (2011) 754-770.

[5] F. Hoffmann, M. Cornelius, J. Morell, M. Fröba, Angew. Chem. Int. Ed. 45 (2006) 3216-3251.

[6] G.L. Athens, R.M. Shayib, B.F. Chmelka, Curr. Opin. Colloid Interface Sci. 14 (2009) 281-292.

[7] M. Keppeler, N. Hüsing, New J. Chem. 35 (2011) 681-690.

[8] A. Sachse, A. Galarneau, F. Fajula, F. Di Renzo, P. Creux, B. Coq, Microporous Mesoporous Mater. 140 (2011) 58-68.

[9] M. Keppeler, J. Holzbock, J. Akbarzadeh, H. Peterlik, N. Hüsing, J. Ceram. Soc. Jpn. 123 (2015) 764-769.

[10] K. Nakanishi, N. Soga, J. Am. Ceram. Soc. 74 (1991) 2518-2530.

[11] K. Nakanishi, J. Porous Mater. 4 (1997) 67-112.

[12] K. Nakanishi, R. Takahashi, T. Nagakane, K. Kitayama, N. Koheiya, H. Shikata, N. Soga, J. Sol-Gel Sci. Technol. 17 (2000) 191-210.

[13] D. Hlushkou, S. Bruns, A. Seidel-Morgenstern, U. Tallarek, J. Sep. Sci. 34 (2011) 2026-2037.

[14] G. Guiochon, J. Chromatogr. A 1168 (2007) 101-168.

[15] K.K. Unger, R. Skudas, M.M. Schulte, J. Chromatogr. A 1184 (2008) 393-415.

[16] A. Galarneau, A. Sachse, B. Said, C.-H. Pelisson, P. Boscaro, N. Brun, L. Courtheoux, N. Olivi-Tran, B. Coasne, F. Fajula, C. R. Chim. 19 (2016) $231-247$.

[17] A. Sachse, A. Galarneau, B. Coq, F. Fajula, New J. Chem. 35 (2011) 259-264.

[18] T. Müllner, K.K. Unger, U. Tallarek, New J. Chem. 40 (2016) 3993-4015.

[19] D. Enke, R. Gläser, U. Tallarek, Chem. Ing. Tech. 88 (2016) 1561-1585.

[20] J.-H. Smått, C. Weidenthaler, J.B. Rosenholm, M. Lindén, Chem. Mater. 18 (2006) 1443-1450.

[21] Y.-S. Hu, P. Adelhelm, B.M. Smarsly, S. Hore, M. Antonietti, J. Maier, Adv. Funct. Mater. 17 (2007) 1873-1878.

[22] J.-H. Smått, F.M. Sayler, A.J. Grano, M.G. Bakker, Adv. Eng. Mater. 14 (2012) 1059-1073.

[23] T. Hara, S. Mascotto, C. Weidmann, B.M. Smarsly, J. Chromatogr. A 1218 (2011) 3624-3635.

[24] N. Hüsing, U. Schubert, Angew. Chem. Int. Ed. 37 (1998) 22-45.

[25] N. Hüsing, F. Schwertfeger, W. Tappert, U. Schubert, J. Non-Cryst. Solids 186 (1995) 37-43.

[26] B.M. Gauthier, S.D. Bakrania, A.M. Anderson, M.K. Carroll, J. Non-Cryst. Solids 350 (2004) 238-243.

[27] L.S. White, M.F. Bertino, G. Kitchen, J. Young, C. Newton, R. Al-Soubaihi, S. Saeed, K. Saoud, J. Mater. Chem. A 3 (2015) 762-772.

[28] L.S. White, M.F. Bertino, S. Saeed, K. Saoud, Microporous Mesoporous Mater. 217 (2015) 244-252.

[29] R. Miyamoto, Y. Ando, C. Kurusu, H. Bai, K. Nakanishi, M. Ippommatsu, J. Sep. Sci. 36 (2013) 1890-1896.

[30] S. Yoda, S. Ohshima, J. Non-Cryst. Solids 248 (1999) 224-234.

[31] K. Hormann, U. Tallarek, J. Chromatogr. A 1312 (2013) 26-36.

[32] T. Müllner, A. Zankel, Y. Lv, F. Svec, A. Höltzel, U. Tallarek, Adv. Mater. 27 (2015) 6009-6013.

[33] M. Thommes, K.A. Cychosz, Adsorption 20 (2014) 233-250.

[34] K. Hormann, T. Müllner, S. Bruns, A. Höltzel, U. Tallarek, J. Chromatogr. A 1222 (2012) 46-58.

[35] D. Hlushkou, K. Hormann, A. Höltzel, S. Khirevich, A. Seidel-Morgenstern, U. Tallarek, J. Chromatogr. A 1303 (2013) 28-38.

[36] K. Hormann, V. Baranau, D. Hlushkou, A. Höltzel, U. Tallarek, New J. Chem. 40 (2016) 4187-4199.

[37] D. Stoeckel, C. Kübel, M.O. Loeh, B.M. Smarsly, U. Tallarek, Langmuir 31 (2015) 7391-7400. 